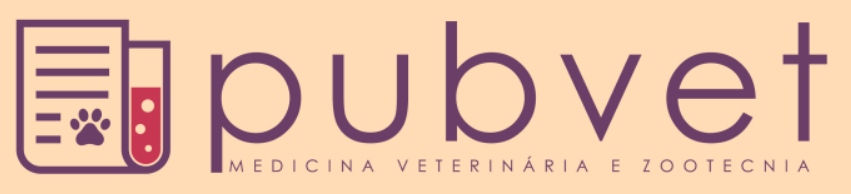

https://doi.org/10.22256/pubvet.v12n4a76.1-5

\title{
Avaliação do crescimento e desempenho de diferentes linhagens de frango caipira melhorado na região de goianésia Goiás
}

\author{
Alan Vitor de Lemos ${ }^{1}$, Dyb Youssef Bittar ${ }^{\bullet} 2 *$, Oscar Vitor Neto ${ }^{\bullet}$, Wagner Gonçalves \\ Vieira Junior 1
}

'Discente do curso de agronomia, Faculdade Evangélica de Goianésia, Brasil.

${ }^{2}$ Docente do Curso de Agronomia, Faculdade Evangélica de Goianésia, Brasil

*Autor para correspondência, E-mail: dybittar@hotmail.com

\begin{abstract}
RESUMO. Dentro da avicultura um dos segmentos que tem apresentado maior crescimento, é a criação de aves para a produção de carne tipo caipira, que visa atender a um grupo mais exigente de consumidores. Esse crescimento proporcionou um aumento da produção de aves criadas em sistemas alternativos nos últimos anos. E visa conseguir atender aos interesses dos consumidores que buscam por carnes com características diferenciadas das aves criadas convencionalmente. Objetivou-se com a pesquisa comparar o potencial de crescimento e o desempenho zootécnico com ênfase na conversão alimentar de quatro linhagens de frangos, para produção de carne tipo caipira, sendo as linhagens Carijó (CA), Mesclado (MS), Pescoço Pelado (PP) e Pesadão (PS). O delineamento experimental utilizado foi em blocos casualizado com parcelas subdivididas, sendo 4 tratamentos e cinco repetições. O experimento foi conduzido em galpão convencional com as medidas de $15 \mathrm{~m} \mathrm{X} 6 \mathrm{~m}$, com o solo coberto com cama vegetal, composta com casca de arroz na espessura de $10 \mathrm{~cm}$ de altura. Observou que a linhagem Pescoço Pelado (PP) obteve uma melhor conversão alimentar e melhor adaptabilidade ao ambiente.
\end{abstract}

Palavras chave: aves, criação alternativa, conversão alimentar, índices zootécnicos

\section{Evaluation of growth and performance of different lines of country chicken improved in goianésia Goiás region}

ABSTRACT. Within the poultry industry, one of the segments that has shown the greatest growth is poultry breeding for the production of "caipira" meat, which aims to serve a more demanding group of consumers his growth has led to an increase in poultry production in alternative systems in recent years. It aims to meet the interests of consumers looking for meats with differentiated characteristics of conventionally created birds. The objective of this research was to compare growth potential and performance with emphasis on the feed conversion of four strains of broiler chickens, for Carijó (CA), Mesclado (MS), Neck Peeled (PP) and Pesadão (PS). The experimental design a randomized block with subdivided plots, four treatments and five replications. They were conducted in a conventional shed with the measurements of $15 \mathrm{~m} \mathrm{X} 6 \mathrm{~m}$, with the soil covered with vegetal bed, composed with rice husk in the thickness of $10 \mathrm{~cm}$ in height. He observed that the Breed Neck (PP) obtained a better-feed conversion and better adaptability to the environment.

Keywords: Poultry, alternative breeding, feed conversion, zootechnical indexes 


\title{
Avaluación del crecimiento y desempeño de diferentes linajes de pollo campesino mejorado en la región de goianésia Goiás
}

\begin{abstract}
RESUMEN. Dentro de la avicultura uno de los segmentos que ha presentado mayor crecimiento, es la cría de aves para producción de carne tipo campesino, que pretende atender a un grupo más exigente de consumidores. Este crecimiento proporcionó un aumento de la producción de aves criadas en sistemas alternativos en los últimos años. Y pretende lograr atender a los intereses de los consumidores que buscan carnes con características diferenciadas de las aves criadas convencionalmente. Se objetivó con esta investigación comparar el potencial de crecimiento y el rendimiento zootécnico con énfasis en la conversión de alimentos de cuatro linajes de pollos para producción de carne tipo campesina, siendo las razas Carijó (CA), Mezclado (MS), Cuello Pelado (PP) y Pesadón (PS). El delineamiento experimental utilizado fue en bloques al azar con parcelas subdivididas, siendo 4 tratamientos y cinco repeticiones. El experimento fue conducido en galpón convencional con las medidas de $15 \mathrm{~m} \mathrm{X} 6 \mathrm{~m}$, con suelo cubierto con cama vegetal, compuesta de cascarilla de arroz con espesor de $10 \mathrm{~cm}$ de altura. Se observó que la raza Cuello Pelado (PP) obtuvo una mejor conversión alimenticia y mejor adaptabilidad al ambiente.
\end{abstract}

Palabras clave: aves, creación alternativa, conversión alimenticia, índices zootécnicos

\section{Introdução}

Dentro da avicultura um dos segmentos que tem apresentado maior crescimento é a criação de aves para a produção de carne tipo caipira, que visa atender a um grupo mais exigente de consumidores (Santos et al., 2005). Esse crescimento proporcionou um aumento da produção de aves criadas em sistemas alternativos nos últimos anos. E visa conseguir atender aos interesses dos consumidores que buscam por carnes com características diferenciadas das aves criadas convencionalmente (Dourado et al., 2009).

As aves melhoradas, quando criadas de forma intensiva, semi-intensiva, ou extensivas fazem com que os produtores tenham animais com bons desempenhos, além de produtos para oferecer ao mercado, com sabor característico dos produtos caipiras. Um método que chamamos de "Sistema Caipira de Criação de Aves" (Ferreira et al., 2014). $\mathrm{O}$ sistema semi-intensivo de produção de aves caipira para corte é dividido por fases, de acordo com a idade, as quais resultam em mudanças fisiológicas e em diferentes necessidades nutricionais; a saber: Fase I - Inicial (01 a 28 dias); Fase II - Crescimento (29 a 56 dias); e Fase Final - Engorda (57 dias até o abate) (Oliveira et al., 2003);

Para que o produtor realize o planejamento da atividade visando a lucratividade é importante que ele conheça o desempenho das aves e também a necessidade das mesmas. Sendo assim é necessário realizar a avaliação das linhagens comerciais que estão atualmente disponíveis para criação em sistema confinado. Para aumentar a lucratividade desse sistema de produção, é importante obter informações relacionadas ao crescimento, desempenho, rendimento e qualidade de carcaça dessas linhagens (Dourado et al., 2009).

O objetivo geral desta pesquisa foi comparar o potencial de crescimento e o desempenho zootécnico com ênfase na conversão alimentar de quatro linhagens de frangos, para produção de carne tipo caipira, sendo as linhagens Carijó, Mesclado, Pescoço Pelado e Pesadão, esperando o resultado especifico de qual dessas linhagens pode responder melhor nos índices zootécnicos, para serem recomendas para produtores da região de estudo e assim alcançar maiores retornos econômicos.

\section{Material e Métodos}

O experimento foi implantado e conduzido no período de 11 de janeiro de 2017 a 21 de março 2017 na Fazenda Laranjal, localizada na Rodovia GO 338, S/N km 43, Município de Pirenópolis Goiás. Caracterizada pelas seguintes coordenadas cartesianas: latitude Sul: $15^{\circ} 36 ' 15.62 " \mathrm{~S}$, longitude Oeste: $49^{\circ} 7{ }^{\prime} 50.40 " 0$, altitude aproximada de 341 m. O clima do local, segundo a classificação de Köppen \& Geiger (1928) é do tipo Aw (quente e seco com estação bem definida, de maio a setembro), tropical semi úmido. As temperaturas mínimas geralmente ficam em torno de $14^{\circ} \mathrm{C}$ e as máximas alcançam acima dos 34 C. A 
precipitação anual é de cerca de $1575 \mathrm{~mm}$. O galpão utilizado está disposto no sudeste-nordeste com as seguintes dimensões $15 \mathrm{~m} \times 6 \mathrm{~m}$ e pé direito de $2,70 \mathrm{~m}$. O galpão possui muretas laterais de 30 $\mathrm{cm}$ de altura e piso de concreto, cobertura em telha de amianto 3,66 x 1,10 m, cortinas e telas até o teto. Foi utilizado somente $20 \mathrm{~m}^{2}$ do galpão; porém todo o galpão foi isolado somente para a realização do experimento. A cama de cobertura utilizada para cobrir o solo, evitando danos a carcaça e melhorando a ambiência para a produção foi de casca de arroz, espalhada homogeneamente com espessura de $10 \mathrm{~cm}$ de altura.

Foram utilizadas 200 aves, sendo 50 aves da linhagem Carijó (CA), 50 aves da linhagem Mesclado (MS), 50 aves da linhagem Pescoço Pelado (PP) e 50 aves da linhagem Pesadão (PS). $\mathrm{O}$ estudo foi realizado no sistema de criação intensivo. As aves foram distribuídas em um delineamento experimental em blocos casualizados, com 4 tratamentos e cinco repetições contendo 10 aves cada. Os tratamentos consistiram em avaliar as linhagens (CA, MS, PP e PS), em seus desempenhos de ganho de peso, conversão alimentar e observando os índices zootécnicos para comparativo de desempenho entre elas. As aves foram adquiridas no município de Goiânia - Goiás. Na recepção foi realizado: pesagem individual, vistoria e análise de defeitos genéticos, avaliação da taxa de mortalidade e distribuídos por sorteio para cada grupo pertencente. $\mathrm{Na}$ fase inicial foi direcionado diretamente para o galpão de produção e separados em grupos de avaliação com 10 indivíduos cada com 20 repetições. A ração foi fornecida em comedouros tubulares de $5 \mathrm{~kg}$ para todas as fases com consumo a vontade, e a água em bebedouros pendulares de 5 litros.

A iluminação foi realizada por lâmpadas incandescente de $60 \mathrm{wtz}$ em todo o galpão, durante o período noturno, para que incentivasse os indivíduos a aumentar o consumo alimentar durante a noite tendo assim melhor aproveitamento de tempo. As aves foram alimentadas com rações formuladas para atender às exigências nutricionais das mesmas, conforme programa nutricional recomendado para cada fase. A reposição da ração foi realizada uma vez por dia com pesagem diária da quantidade fornecida e consumida. O programa alimentar adotado foi escolhido de acordo com a idade das aves, sendo: inicial de 1 a 21 dias, crescimento de 22 a 45 dias, e a fase final de 46 a 70 dias. A duração total do experimento foi de 70 dias, sendo do dia $11 / 01 / 2017$ ao 21/03/2017, separando as fases e a duração conforme o programa alimentar.

A limpeza dos bebedouros foi feita duas vezes por dia sendo no período da manhã e tarde durante a reposição de água. A cama de frango formada por casca de arroz era trocada quando a umidade aumentava, para que houvesse a redução da proliferação de microrganismos patogênicos. As aves foram pesadas a cada 7 dias e a ração diariamente para determinação do ganho de peso (g/ave), do consumo de ração (g/ave) e da conversão alimentar $(\mathrm{g} / \mathrm{g})$. Os dados foram avaliados aos 21, 46 e 70 dias. Os resultados obtidos nas avaliações das quatro linhagens foram submetidos à análise de variância (teste $\mathrm{F}$ ) e as médias foram comparadas pelo teste de Tukey a $5 \%$ de probabilidade de erro, utilizando o programa de estatística de Ferreira (2011).

\section{Resultados e Discussão}

Na tabela 1 consta a comparação de médias do efeito de genótipo para a variável consumo de ração diária e total nas fases inicial, crescimento e terminação respectivamente.

Para consumo de ração diário na fase inicial e crescimento houve diferença $(\mathrm{P}<0,05)$. Os animais da linhagem CA, MS e PS foram superiores seguidos pelo PP. Na fase terminação não houve diferença $(\mathrm{P}>0,05)$ no consumo de ração diário.

Tabela 1. Consumo de ração ao dia (CRD) e total (CRT) de diferentes linhagens de frango caipira

\begin{tabular}{lcccccc}
\hline \multirow{2}{*}{ linhagem } & \multicolumn{2}{c}{ Inicial } & \multicolumn{2}{c}{ Crescimento } & \multicolumn{2}{c}{ Terminação } \\
\cline { 2 - 7 } & CRD & CRT & CRD & CRT & CRD & CRT \\
\hline Carijó & $9.43 \mathrm{a}$ & $217.98 \mathrm{a}$ & $102.72 \mathrm{a}$ & $2467.00 \mathrm{a}$ & $151.80 \mathrm{a}$ & $3795.00 \mathrm{a}$ \\
Mesclado & $9.29 \mathrm{a}$ & $229.74 \mathrm{a}$ & $107.00 \mathrm{a}$ & $2577.80 \mathrm{a}$ & $151.40 \mathrm{a}$ & $3785.00 \mathrm{a}$ \\
Pescoço Pelado & $9.07 \mathrm{a}$ & $126.00 \mathrm{c}$ & $86.40 \mathrm{~b}$ & $2075.80 \mathrm{~b}$ & $160.80 \mathrm{a}$ & $4020.00 \mathrm{a}$ \\
Pesadão & $8.73 \mathrm{a}$ & $183.12 \mathrm{~b}$ & $97.600 \mathrm{a}$ & $2347.00 \mathrm{a}$ & $153.00 \mathrm{a}$ & $3825.00 \mathrm{a}$ \\
\hline
\end{tabular}

As médias seguidas pela mesma letra na coluna não diferem estatisticamente entre si. Foi aplicado o Teste de Tukey ao nível de $5 \%$ de probabilidade. 
Na avaliação do consumo de ração total na fase inicial e crescimento houve diferença $(\mathrm{P}<0,05)$, sendo os animais da linhagem CA e MS foram superiores seguidos pelo PS e PP. Na fase de crescimento as linhagens CA, MS e PS foram superiores ao PP. Na fase terminação não houve diferença significativa.

Hellmeister Filho et al. (2003) e Carrijo et al. $\underline{(2010)}$ ao avaliarem linhagens alternativas na criação de frango tipo caipira encontraram diferenças entre linhagens para consumo de ração; porém não verificaram diferenças entre linhagens para conversão alimentar. Desta forma, podemos concordar que os dados obtidos na pesquisa corroboram com os dados de Hellmeister Filho et al., (2003) e Carrijo et al., (2010) para consumo de ração nas fase inicial e crescimento; porém não obtivemos o mesmo resultado na fase de terminação pois não houve diferenças estatísticas significativas.

Na tabela 2 estão descritos o ganho de peso diário e total entre as diferentes linhagens, avaliados durante o experimento.

Tabela 2. Ganho de peso diário (GPD) e ganho de peso total (GPT) de diferentes linhagens de frango caipira

\begin{tabular}{lcccccc}
\hline \multirow{2}{*}{ Linagens } & \multicolumn{2}{c}{ Inicial } & \multicolumn{2}{c}{ Crescimento } & \multicolumn{2}{c}{ Terminação } \\
\cline { 2 - 7 } & GPD & GPT & GPD & GPT & GPD & GPT \\
\hline Carijó & $12.80 \mathrm{a}$ & $268.80 \mathrm{a}$ & $63.20 \mathrm{a}$ & $1516.80 \mathrm{a}$ & $52.80 \mathrm{c}$ & $1320.00 \mathrm{c}$ \\
Mesclado & $10.80 \mathrm{~b}$ & $226.80 \mathrm{~b}$ & $61.60 \mathrm{a}$ & $1478.40 \mathrm{a}$ & $55.00 \mathrm{~b}$ & $1375.00 \mathrm{~b}$ \\
Pescoço Pelado & $9.54 \mathrm{c}$ & $200.34 \mathrm{c}$ & $46.60 \mathrm{~b}$ & $1118.40 \mathrm{~b}$ & $71.80 \mathrm{a}$ & $1795.00 \mathrm{a}$ \\
PS & $7.22 \mathrm{~d}$ & $151.62 \mathrm{~d}$ & $45.20 \mathrm{~b}$ & $1084.80 \mathrm{~b}$ & $73.00 \mathrm{a}$ & $1825.00 \mathrm{a}$ \\
\hline
\end{tabular}

As médias seguidas pela mesma letra não diferem estatisticamente entre si. Foi aplicado o Teste de Tukey ao nível de 5\% de probabilidade

Em relação ao ganho de peso diário na fase inicial houve diferença $(\mathrm{P}<0,05)$. A linhagem $\mathrm{CA}$ foi superior aos demais seguidas respectivamente pelas linhagens MS, PP e PS. Na fase de crescimento os animais da linhagem CA e MS foram superiores $(\mathrm{P}<0,05)$ aos demais. $\mathrm{Na}$ fase de terminação houve diferença $(\mathrm{P}<0,05)$. As linhagens PP e PS foram superiores as demais seguidas pelas linhagens MS e CA. Santos \& Grangeiro (2014) avaliando e diferentes linhagens de frangos caipiras, encontraram resultados semelhantes ao trabalho. No entanto, Silva et al. (2003) avaliando ganho de peso e conversão alimentar em aves criadas em semi confinamento encontraram valores melhores em relação ao peso corporal e conversão alimentar e relatam ainda que o sistema de semi confinamento proporciona bem-estar às aves e, por isso, influenciou positivamente no desempenho. Santos et al. (2005) também verificaram maior ganho de peso no sistema de semi confinamento, devido ao melhor conforto das aves e bem-estar.

Podemos observar que houve mudanças no ganho peso/fase sendo que as linhagens CA e MS obtiveram maior ganho de peso nas fases inicial e crescimento; porém na fase de terminação os melhores resultados foram das linhagens PP e PS, assim podemos observar que há um gargalo que pode ser avaliado, como diferentes dias de abate para cada linhagem analisada no experimento.
Em trabalhos realizados por Santos et al. (2005), a taxa de crescimento de machos de linhagens caipiras começa a se diferenciar a partir do $28^{\circ}$ dia, apresentando crescimento mais acelerado, com melhor eficiência de utilização do alimento e consequentemente, melhores conversões.

Devido ao intenso melhoramento genético as aves têm sido selecionadas para aumentar a taxa de crescimento, como consequência ocorre o aumento de consumo de alimentos o que melhora a eficiência e antecipa a idade de abate destes animais de acordo com (Pym, 2005).

$\mathrm{Na}$ tabela 3 conseguimos visualizar os dados, quanto a conversão alimentar nas fases: inicial, crescimento e terminação.

Tabela 3. Conversão alimentar fases: inicial, crescimento e terminação de diferentes linhagens de frango caipira

\begin{tabular}{lccc}
\hline \multirow{2}{*}{ Linhagem } & \multicolumn{3}{c}{ Conversão alimentar } \\
\cline { 2 - 4 } & Inicial & Crescimento Terminação \\
\hline Carijó & $0.81 \mathrm{c}$ & $1.62 \mathrm{c}$ & $2.87 \mathrm{a}$ \\
Mesclado & $1.01 \mathrm{~b}$ & $1.74 \mathrm{bc}$ & $2.75 \mathrm{a}$ \\
Pescoço Pelado & $0.62 \mathrm{~d}$ & $1.85 \mathrm{~b}$ & $2.24 \mathrm{~b}$ \\
PS & $1.21 \mathrm{a}$ & $2.17 \mathrm{a}$ & $2.09 \mathrm{~b}$ \\
\hline
\end{tabular}

As médias seguidas pela mesma letra não diferem estatisticamente entre si. Foi aplicado o Teste de Tukey ao nível de $5 \%$ de probabilidade. 
Podemos observar na tabela 3 , que as aves da linhagem pescoço pelado (PP) apresentaram melhor resultado na conversão alimentar referente às outras linhagens, sendo assim teve menor consumo de ração e maior ganho de peso, o que nos mostra seu melhor desempenho na conversão alimentar, assim conseguimos avaliar que entre as quatro linhagens submetidas na pesquisa, o PP (Pescoço Pelado), obteve melhor resultados comparados com todas as outras, tendo diferenças significativas.

\section{Conclusão}

As aves da linhagem Pescoço Pelado apresentaram valores melhores em relação ao peso corporal e conversão alimentar destacandose positivamente na eficiência de utilização do alimento.

\section{Referências Bibliográficas}

Carrijo, A. S., Fascina, V. B., Souza, K. M. R., Ribeiro, S. S., Allaman, I. B., Garcia, A. M. L. \& Higa, J. A. 2010. Níveis de farelo da raiz integral de mandioca em dietas para fêmeas de frangos caipiras. Revista Brasileira de Saúde e Produção Animal, 11, 131-139.

Dourado, L. R. B., Sakomura, N. K., Nascimento, D. C. N. d., Dorigam, J. C., Marcato, S. M. \& Fernandes, J. B. K. 2009. Crescimento e desempenho de linhagens de aves pescoço pelado criadas em sistema semi-confinado. Ciência e Agrotecnologia, 33, 875-881.

Ferreira, D. F. 2011. SISVAR: A Computer Statistical Analysis System. Ciência $e$ Agrotecnologia, 35, 1039-1042.

Ferreira, M. W., Marques, R. R., Abreu, A. P. N. \& Silva, T. R. 2014. Desempenho de frangos caipiras Label Rouge alimentados com farelo de amendoim em substituição parcial ao farelo de soja. Revista Brasileira de Ciência Veterinária, 21, 105-109.

Hellmeister Filho, P., Menten, J. F. M., Silva, M. A. N., Coelho, A. A. D. \& Savino, V. J. M. 2003. Efeito de genótipo e do sistema de criação sobre o desempenho de frangos tipo caipira. Revista Brasileira de Zootecnia, 32, 1883-1889.

Köppen, W. \& Geiger, R. 1928. Klimate der Erde. Gotha: Verlag Justus Perthes. Wall-map $150 \mathrm{~cm} \times 200 \mathrm{~cm}$.

Oliveira, J. F., Holanda, J. S. \& Melo, J. B. 2003. Manejo e produção de galinha caipira. Natal: Emparn.

Pym, R. A. E. 2005. Genetic aspects of food intake and food utilisation efficiency for growth in chickens. Proceedings of the 17th Australian Poultry Science Symposium. Poultry Research Foundation, Sydney, New South Wales, Australia.

Santos, A. L., Sakomura, N. K., Freitas, E. R., Fortes, C. M. L. S., Carrilho, E. N. V. M. \& Fernandes, J. B. K. 2005. Estudo do crescimento, desempenho, rendimento de carcaça e qualidade de carne de três linhagens de frango de corte. Revista Brasileira de Zootecnia, 34, 1589-1598.

Santos, J. F. \& Grangeiro, J. I. T. 2014. Desempenho de genótipos de aves caipiras submetidos à dietas com farelo de raízes de mandioca enriquecido com levedura. Tecnologia \& Ciências Agropecuária, 8, 1116.

Silva, M. A. N., Hellmeister Filho, P., Rosario, M. F., Coelho, A. A. D., Savino, V. J. M., Garcia, A. A. F., Silva, I. J. O. \& Menten, J. F. M. 2003. Influência do sistema de criação sobre o desempenho, a condição fisiológica e o comportamento de linhagens de frangos para corte. Revista Brasileira de Zootecnia, 32, 208213.

Article History:

Received 8 December 2017

Accepted 23 January 2018

Available online 2 April 2018

License information: This is an open-access article distributed under the terms of the Creative Commons Attribution License 4.0, which permits unrestricted use, distribution, and reproduction in any medium, provided the original work is properly cited. 